\title{
Age differences in postoperative pain are scale dependent: a comparison of measures of pain intensity and quality in younger and older surgical patients
}

\author{
Lucia Gagliese $e^{\mathrm{a}, \mathrm{b}, \mathrm{c}, *}$, Joel Katz ${ }^{\mathrm{a}, \mathrm{c}, \mathrm{d}}$ \\ ${ }^{a}$ School of Kinesiology and Health Science, York University, Toronto, Ontario, Canada \\ ${ }^{\mathrm{b}}$ Department of Psychology, York University, Toronto, Ontario, Canada

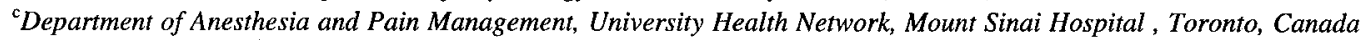 \\ ${ }^{\mathrm{d}}$ Department of Anesthesia, University of Toronto, Toronto, Ontario, Canada
}

\begin{abstract}
As the population ages, research into the assessment of postoperative pain in older patients is urgently needed. The reliability and validity of most pain scales for the assessment of acute postoperative pain in the elderly remain to be demonstrated. The present study reports the analysis of age-related patterns on three pain scales (McGill Pain Questionnaire, MPQ; Present Pain Intensity, PPI; and Visual Analog Scale, VAS) completed by younger $(n=95$, mean age $=56.4 \pm 5.8$ years $)$ and older $(n=105$; mean age $=66.8 \pm 2.7$ years $)$ men following radical prostatectomy. All patients received intravenous morphine via patient-controlled analgesia (PCA) throughout the study. On the first 2 postoperative days (POD), patients completed the pain scales and PCA opioid intake was recorded. An interaction was found between amount of opioid self-administered and POD. In both groups, less opioid was administered on POD 2 than POD 1, but the decrease over time was greater in younger than older men. On both PODs, older men self-administered less opioid than younger men. Age differences in pain were dependent on the pain scale used. Older men had significantly lower scores than younger men on the MPQ and PPI but there were no differences on the VAS. Several age differences in the psychometric properties of the scales were evident. On both PODs, the correlation between VAS and MPQ scores was significantly lower in the older than younger group. POD effect sizes did not differ between the scales or age groups suggesting that all three scales have comparable sensitivity within an age group. However, the different results between the scales for the effect of age suggests that the VAS is not sufficiently sensitive to detect age differences. Therefore, age differences in postoperative pain are better captured by verbal descriptions of pain qualities than non-verbal measures of intensity.
\end{abstract}

Keywords: Postoperative pain; Psychometrics; McGill Pain Questionnaire; Opioids; Patient-controlled analgesia; Geriatrics

\section{Introduction}

As the population ages (U.S. Department of Commerce, 1991), the number of elderly people undergoing surgical procedures will increase (Politser and Schneidman, 1990). Similar to younger patients, these people will require effective and safe postoperative pain control. As such, research into the assessment and management of postoperative pain in older patients is urgently needed. Effective management of pain is dependent upon valid and reliable assessment (Turk and Melzack, 2001). The key role of assessment has been recognized by its adoption as the fifth vital sign in the postoperative period (American Pain Society, 1995). Effec-

\footnotetext{
* Corresponding author. Tel.: +1-416-340-4296; fax: +1-416-340-4739.

E-mail address: lucia.gagliese@uhn.on.ca (L. Gagliese).
}

tive management of acute postoperative pain is essential, in part, because unrelieved postoperative pain has serious immediate and long term consequences. In the immediate postoperative period, unrelieved pain is associated with respiratory, renal and cardiac dysfunction or failure (Cousins, 1994), immune suppression (Ergina et al., 1993), and delirium (Duggleby and Lander, 1994). In the long term, unrelieved postoperative pain is associated with functional impairment (Pasero and McCaffery, 1996a,b) and the development of chronic postsurgical pain (Katz, 1997). The fundamental issue of how best to measure pain in elderly surgical patients and across age groups has yet to be resolved in spite of the anticipated increase in the number of elderly surgical patients and the recognition of the importance of effective pain management.

The most commonly employed pain scales were devel- 
oped for younger adults, and their psychometric properties when used with elderly people have yet to be adequately demonstrated (Gagliese, 2001). Several preliminary reports have suggested that verbal descriptor and numeric scale measures of intensity-and the McGill Pain Questionnaire (MPQ), a measure of the qualities of pain (Melzack, 1975), may have adequate to good reliability and validity for the assessment of chronic pain in the elderly (Herr and Mobily, 1993; Lewis et al., 1995; Benesh et al., 1997; Gagliese and Melzack, 1997). In contrast, problems have been reported with the use of visual analog scales (VAS) by elderly people, including different intensity estimates from those obtained on other measures and difficulties understanding and completing the scale (Kremer et al., 1981; Jensen et al., 1986; Herr and Mobily, 1993; Gagliese and Melzack, 1997). Reasons for these difficulties remain to be elucidated. Unfortunately, firm conclusions cannot be drawn from these studies due to methodological variability, small sample sizes, and lack of age comparisons.

Importantly, the reliability and validity of these scales for the assessment of acute postoperative pain in the elderly remain to be demonstrated. This population is distinct from those with chronic pain in several respects. The most important differences, especially among the elderly, may be the effect of factors such as recovery from anaesthesia, acute opioid administration, acute illness, and the destabilizing effects of the novel hospital environment. Each of these factors may influence scale comprehension and completion and hence the reliability and validity of the measures. As a result, it may not be appropriate to generalize data from samples of chronic pain patients to those with postoperative pain (Dunn, 1989). Given the rapidly increasing numbers of elderly surgical patients and the serious risks associated with unrelieved postoperative pain, it is imperative that the most appropriate pain assessment tools for use with this vulnerable group of patients be identified.

Evidence regarding age differences in postoperative pain levels is equivocal. Although several studies have suggested that elderly patients report lower pain intensity than younger patients (Bellville et al., 1971; Oberle et al., 1990), others have found that age is not related to postoperative pain intensity (Giuffre et al., 1991; Duggleby and Lander, 1994; Gagliese et al., 2000). It is difficult to interpret these results since they may reflect not only age differences in the experience of postoperative pain and analgesic efficacy, but also differences in the validity and reliability of the pain tools across age groups. This highlights the serious barrier to progress in our understanding of aging and pain which arises from lack of psychometric data.

Age-related increases in the analgesic efficacy of opioids have been consistently reported (Kaiko, 1980; Moore et al., 1990). Elderly patients obtain greater analgesia than younger patients in response to a fixed dose of opioids (Bellville et al., 1971; Kaiko, 1980). Also, they self-administer less opioid than young patients but report comparable pain relief using patient-controlled analgesia (PCA) (Burns et al., 1989; Giuffre et al., 1991; Macintyre and Jarvis, 1995; Gagliese et al., 2000). In this modality, patients press a button when they require pain relief to obtain a dose of an analgesic through an intravenous or epidural line (Lehmann, 1991). The apparatus is-set to deliver a fixed dose of the drug with a specified lock-out interval to minimize the possibility of adverse events and accidental overdose (Lehmann, 1991). PCA is associated with good pain control and high satisfaction among younger patients (Egan and Ready, 1994; Perry et al., 1994; Miaskowski et al., 1999). There is growing evidence that PCA also may provide adequate analgesia in elderly patients (Egbert et al., 1990; Macintyre and Jarvis, 1995; Badaoui et al., 1996; Gagliese et al., 2000), with fewer adverse effects than intramuscular injection of opioids (Egbert et al., 1990). The mechanisms for the age differences in opioid effects remain unclear but likely involve various inter-related changes in the pharmacokinetic and pharmacodynamic properties of these drugs with age (Owen et al., 1983; Baillie et al., 1989; Laizure et al., 1993; Van Crugten et al., 1997).

The present study reports the analysis of age-related patterns on three pain scales completed on the first and second day following radical prostatectomy by younger and older men. The specific objectives of the study were to investigate age differences in (i) the psychometric properties of commonly used pain scales for the assessment of postoperative pain, and (ii) analgesic consumption and postoperative pain following radical prostatectomy. This information will be important in the development of pain assessment protocols which are sensitive to the special needs of elderly surgical patients and which are appropriate for use in different age groups.

\section{Methods}

\subsection{Participants}

Men scheduled for radical prostatectomy and who were eligible for postoperative PCA were approached to participate in this study. Patients were excluded if they did not speak or read English sufficiently well to give informed consent or to complete the assessment, if they received an American Society of Anesthesiology score $\geq 3$, weighed more than $100 \mathrm{~kg}$, reported chronic pain of greater than 6 months duration, or chronic use of opioids. Two groups, younger and older, were formed based on a split of the sample at the median age of 62 years. This cut-off for group assignment was chosen in order to maximize the number of patients in each group and not because substantive changes are expected to occur at 62 years of age.

\subsection{Measures}

\subsubsection{McGill Pain Questionnaire (MPQ)}

The MPQ (Melzack, 1975), the most widely used multidimensional pain inventory (Wilke et al., 1990), is made up 
of 20 categories of adjectives that describe the qualities of pain. Subjects are asked to endorse those words that describe their feelings and sensations at that moment. The rank values of the words chosen are summed to obtain a total pain rating index (PRI-T) and separate-scores for the sensory (PRI-S), affective (PRI-A), evaluative (PRI-E), and miscellaneous (PRI-M) subscales. There is substantial evidence for the validity, reliability, sensitivity, and discriminative abilities of the MPQ when used with younger adults (Melzack and Katz, 2001).

\subsubsection{Present pain intensity (PPI)}

The PPI, a part of the MPQ, is a verbal descriptor scale of the intensity of pain ranging from $0=$ none to $5=$ excruciating. Patients are asked to make a mark next to the word that describes their pain 'right now'. Verbal descriptor scales are valid and reliable for younger samples (Jensen and Karoly, 2001) and preliminary evidence supports their use for the assessment of chronic pain in the elderly (Herr and Mobily, 1993; Gagliese and Melzack, 1997).

\subsubsection{Visual analog scale (VAS)}

Intensity of pain at rest (VAS-R) and in response to a standard mobilization exercise (VAS-M) after sitting upright from a lying position and taking two maximal inspirations) were assessed using VAS. The VAS (Huskisson, 1983) is an efficient and minimally intrusive measure of pain intensity that has been used widely in research and clinical settings. It consists of a $10 \mathrm{~cm}$ horizontal line with the two endpoints labeled 'no pain' and 'worst possible pain'. The patient is required to mark the line at a point that corresponds to the level of pain intensity he or she presently feels. The distance in $\mathrm{cm}$ from the low end of the VAS to the mark made by the patient has been shown to be a sensitive, valid, and reliable measure of pain intensity in young samples (Jensen and Karoly, 1992). Concerns regarding use of the VAS for the assessment of pain in the elderly have been expressed although more data are needed before firm conclusions can be drawn (see review by Gagliese, 2001).

\subsection{Procedure}

On the evening before surgery, informed consent was obtained, and patients received instruction in the use of PCA. All patients underwent radical prostatectomy under general anaesthesia. Following surgery, participants were transferred to the post-anaesthetic care unit and were connected to a PCA pump (Abbott Life Care Infuser, Chicago, IL). Participants were asked whether they were in need of pain relief. An affirmative response was followed by a $2-4 \mathrm{mg}$ intravenous (i.v.) bolus of morphine administered by a nurse. This procedure was repeated until the participants were sufficiently alert to begin using the pump on their own. The PCA pump was set to deliver a
1.5-2.0 mg i.v. bolus dose of morphine with a lock-out time of 5-7 min, a maximum dose of $30 \mathrm{mg}$ morphine in any $4 \mathrm{~h}$ period, and no continuous background infusion.

At $24 \pm 2 \mathrm{~h}$ and $48 \pm 2 \mathrm{~h}$ following surgery, patients completed the MPQ, PPI, VAS-R, and VAS-M. Patients' daily PCA opioid intake (in mg morphine) was recorded. This study was approved by the University Health.Network Committee for Research on Human Subjects.

\subsection{Data analysis}

Differences in group characteristics were tested with oneway analysis of variance (ANOVA). Age and time effects on each pain scale and on self-administered opioid were assessed with between-within ANOVA using postoperative day (POD) as the within subjects and age group (AGE) as the between subjects factor.

Age differences in convergent validity and sensitivity were then assessed. Analyses were conducted separately for each age group and each POD. Convergent validity is the extent to which different scales designed to measure the same construct give comparable estimates of that construct (Anastasi, 1988). For instance, scores on the two pain intensity measures, VAS-R and PPI, should be similar when they are completed at the same time. The expected relationship between the MPQ and the intensity measures is less clear because these scales measure different constructs. Specifically, the MPQ assesses the qualities of pain (how it feels) while the PPI and VAS measure intensity (how much it hurts). Regardless, the relationship between the MPQ and the intensity measures should be the same in both age groups. Convergent validity was assessed in two ways. First, interscale correlations were calculated. Because of the large number of correlations calculated, a Bonferroni correction was applied, and $P \leq 0.004$ was required for significance. Age differences in the magnitude of the interscale correlations were tested using Fisher's $Z$ transformation. Second, age differences in the slopes and intercepts of the regression lines relating each pair of scales were tested (Kleinbaum et al., 1988).

Pain scale sensitivity, or ability to detect change, was then assessed. Because postoperative pain is a dynamic state which decreases with time (Melzack et al., 1987), calculation of test-retest reliabilities over a 24-h test interval is inappropriate (Wewers and Lowe, 1990). Instead, the predictable change in pain allows for the assessment of the scales' sensitivity. This was done by comparing the magnitude of change over days (POD effect size) between the age groups (Hunter and Schmidt, 1990; Jensen, 1997).

\section{Results}

\subsection{Patient characteristics}

The groups did not differ in body mass index (BMI), surgical duration, or blood loss (Table 1). Therefore, these 
Table 1

Characteristics of each age group

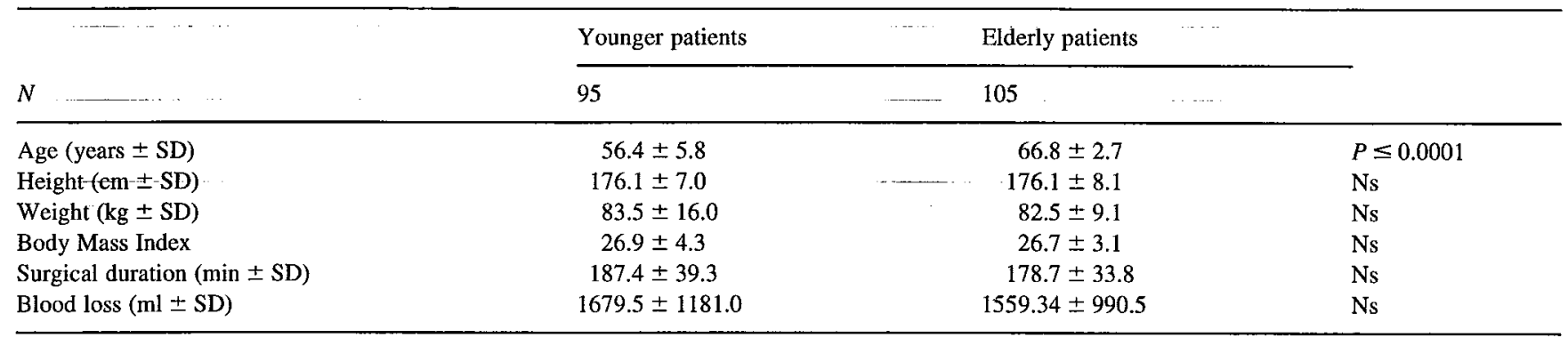

factors were not used as covariates in the subsequent analyses. The average age of the older group is very similar to that of other studies of postoperative pain and aging (eg. Egbert et al., 1990; Duggleby and Lander, 1994; Lynch et al., 1998; Gagliese et al., 2000), supporting our use of the age group criterion and facilitating cross-study comparisons.

\subsection{Age differences in pain scores}

There was a significant effect of AGE $(P \leq 0.009)$ and POD $(P \leq 0.0001)$ on PRI-T. The older patients obtained lower PRI-T scores than the younger patients on both POD 1 $(P \leq 0.03)$ and POD $2(P \leq 0.01)$ (Fig. 1). Detailed analysis of the psychometric properties of the MPQ will be reported separately. There was a significant effect of AGE $(P \leq 0.005)$ and POD $(P \leq 0.01)$ on PPI scores. PPI scores were lower in the older than younger group on POD 1 $(P \leq 0.05)$ and POD $2(P \leq 0.008)$ (Fig. 2). There was no significant effect of AGE on either VAS-R or VAS-M. There was a significant effect of POD on both VAS-R $(P \leq 0.002)$ and VAS-M $(P \leq 0.0001)$ (Fig. 3). On all scales, there was a significant decrease in scores with

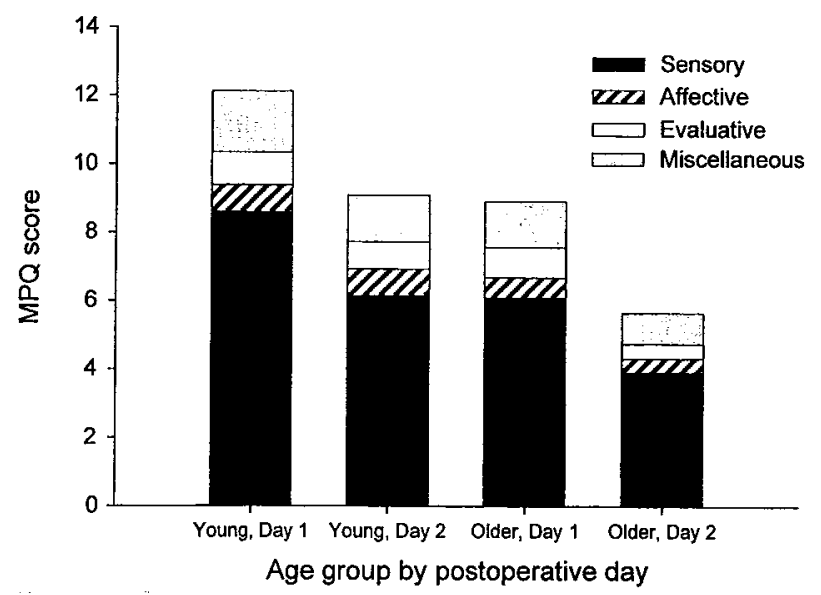

Fig. 1. MPQ scores (PRI-T, rank sum of endorsed descriptors) on POD 1 and POD 2 in younger and older surgical patients. There was a significant effect of AGE $(P \leq 0.009)$ and POD $(P \leq 0.0001)$ on total MPQ scores. The older patients obtained lower PRI-T scores than the younger patients on both POD $1(P \leq 0.03)$ and POD $2(P \leq 0.01)$. time. The interaction between AGE and POD was not significant for any of the analyses.

\subsection{Age differences in PCA opioid use}

There was a significant interaction between AGE and POD $(P \leq 0.01)$ on self-administered opioid (Fig. 4). Older patients self-administered less opioid than younger patients on POD $1(P \leq 0.0001)$ and POD $2(P \leq 0.01)$. The difference between the groups (younger minus older) was greater on POD 1 (13.47 mg) than on POD $2(6.47 \mathrm{mg})$. The amount of morphine self-administered decreased over time in both groups but the decrease (POD 1 minus POD 2) was greater in the younger $(27.68 \pm 22.93 \mathrm{mg})$ than the older $(20.51 \pm 19.58 \mathrm{mg})$ patients $(P \leq 0.02)$.

\subsection{Age differences in the psychometric properties of the pain scales}

\subsubsection{Convergent validity}

There were significant moderate interscale correlations in both age groups with a tendency for the magnitude of the correlations to increase over time, possibly reflecting a practice or learning effect (Table 2). In the younger group, all interscale correlations were significant on both POD 1 and POD 2. In the older group, on POD 1, the correlations between PRI-T and both VAS ratings, and between PPI

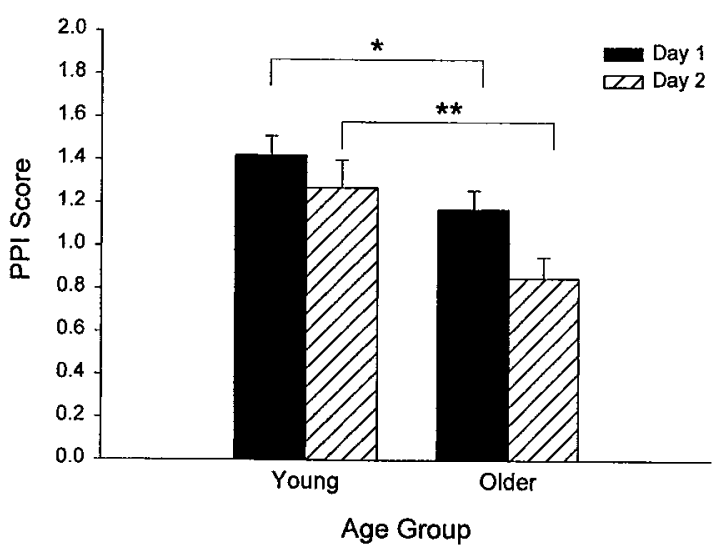

Fig. 2. PPI scores on POD 1 and POD 2 in younger and older surgical patients. There was a significant effect of AGE $(P \leq 0.005)$ and POD $(P \leq 0.01)$ on PPI scores. $* P \leq 0.05 ; * P \leq 0.008$. 


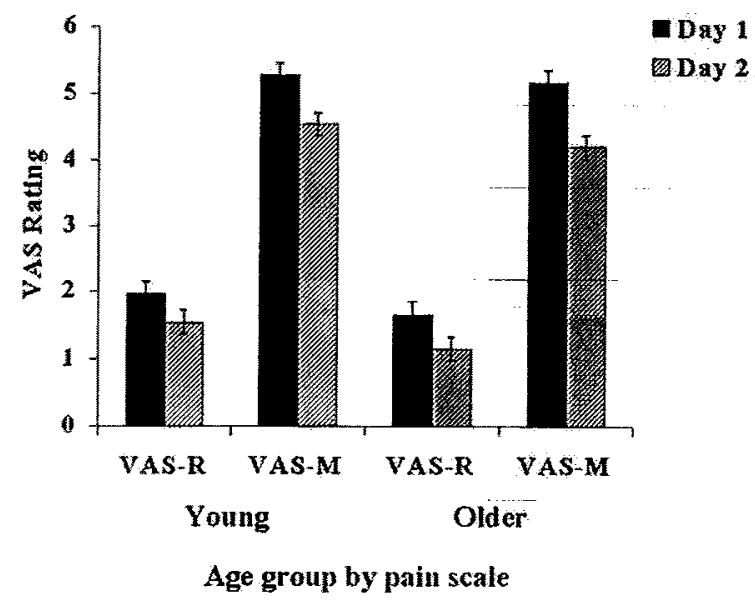

Fig. 3. VAS ratings of pain at rest (VAS-R) and with movement (VAS-M) on POD 1 and POD 2 in younger and older surgical patients. VAS ratings did not differ between the groups. In both age groups, VAS-R $(P \leq 0,002)$ and VAS-M $(P \leq 0.0001)$ decreased with time.

and VAS-M, failed to reach significance. The remaining correlations were significant. On POD 2, the only nonsignificant correlation was between VAS-M and PRI-T. The magnitude of the correlations did not differ between the groups except for VAS-R and PRI-T. On both POD 1 $(P \leq 0.05)$ and POD $2(P \leq 0.004)$, this correlation was lower in older than younger patients.

Fig. 5a-f, which shows the relationship between each pair of scales at each time point, suggests that there is little consistency in estimates across scales. Considerable variability and overlap of scores on different scales was evident in both age groups. Furthermore, there are a large number of discordant pain reports (e.g. scores indicative of mild pain on one scale but intense pain on another). The regression analysis showed that on both POD 1 and POD 2, the relationship between the two measures of intensity (VAS-R and PPI) did not differ between the age groups. The relationship

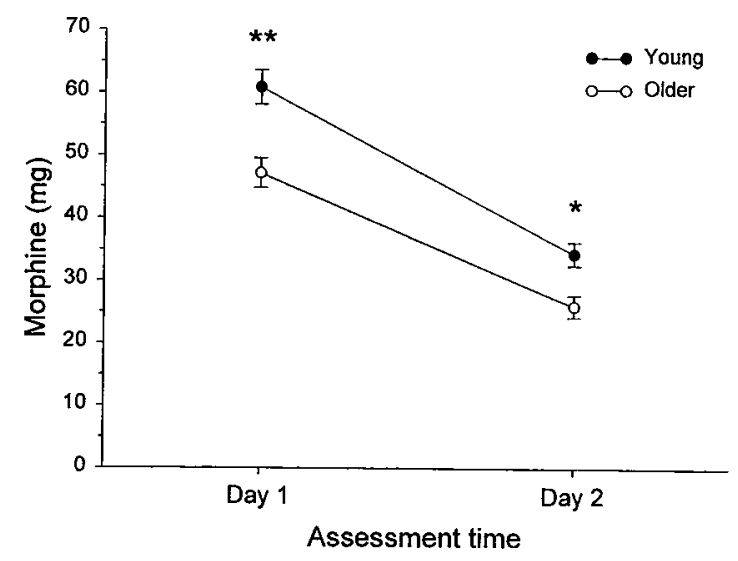

Fig. 4. Daily morphine intake by i.v. PCA in younger and older surgical patients. There was a significant interaction between AGE and POD $(P \leq 0.01)$. Main effect of AGE is shown: $* P \leq 0.012 ; * * P \leq 0.0001$.
Table 2

Pain scale correlations by age groups on POD 1 and POD 2

\begin{tabular}{|c|c|c|c|c|c|c|c|}
\hline & & \multicolumn{2}{|l|}{ MPQ } & \multicolumn{2}{|l|}{ PPI } & \multicolumn{2}{|l|}{ VÁS-R } \\
\hline & & Young & Older & Young & Older & Young & Older \\
\hline \multirow[t]{4}{*}{ Day 1} & MPQ & & & & & & \\
\hline & PPI & $0.54 * *$ & $0.48 * *$ & & - & & \\
\hline & VAS-R & $0.48^{* *}$ & 0.27 & $0.43^{* *}$ & $0.52 * *$ & & \\
\hline & VAS-M & $0.32^{*}$ & 0.17 & $0.38^{*}$ & 0.25 & $0.55^{* *}$ & $0.45^{* *}$ \\
\hline \multicolumn{8}{|l|}{ Day 2} \\
\hline & MPQ & & & & & & \\
\hline & PPI & $0.73 * *$ & $0.79 * *$ & & & & \\
\hline & VAS-R & $0.70 * *$ & $0.44 * *$ & $0.68 * *$ & $0.62 * *$ & & \\
\hline & VAS-M & $0.31 *$ & 0.29 & $0.36^{*}$ & $0.38 *$ & $0.56^{* *}$ & $0.54^{* *}$ \\
\hline
\end{tabular}

$* P \leq 0.004 ; * P \leq 0.0001$.

between VAS-R and PRI-T was different in young and older patients on POD $1(P \leq 0.05)$ and POD $2(P \leq 0.05)$.

\subsubsection{Pain scale sensitivity}

The POD effect sizes were similar across scales and age groups (range of effect size differences $=0.16$ ) (Table 3 ). This suggests that the sensitivity of each scale was comparable both within and across the age groups. The largest effect size was found on PRI-T, followed by the intensity measures. The small effect sizes are not surprising given that all patients had access to opioids on both days. As a result, pain levels were kept fairly low, limiting the magnitude of change over time.

\section{Discussion}

On the first 2 days following radical prostatectomy, older men self-administered less opioid than younger men using PCA. However, age differences in pain were dependent on the assessment instrument used. Specifically, scores on the MPQ and PPI suggested decreased pain or improved analgesia with age whereas VAS scores suggested no difference with age. These results were not secondary to age differences in surgical procedure, tissue damage (surgical duration and blood loss are often considered surrogate measures of tissue damage (Bennett-Guerrero et al., 1999; Gagliese et al., 2000)), or BMI, which did not differ between the groups. The pain scale discrepancy may reflect age differences in the psychometric properties of these scales or age-related changes in the experience of pain.

Table 3

Effect size of change over days for each pain scale by age group

\begin{tabular}{lll}
\hline & Younger & Older \\
\hline MPQ & 0.31 & 0.39 \\
PPI & 0.25 & 0.26 \\
VAS-R & 0.23 & 0.32 \\
\hline
\end{tabular}




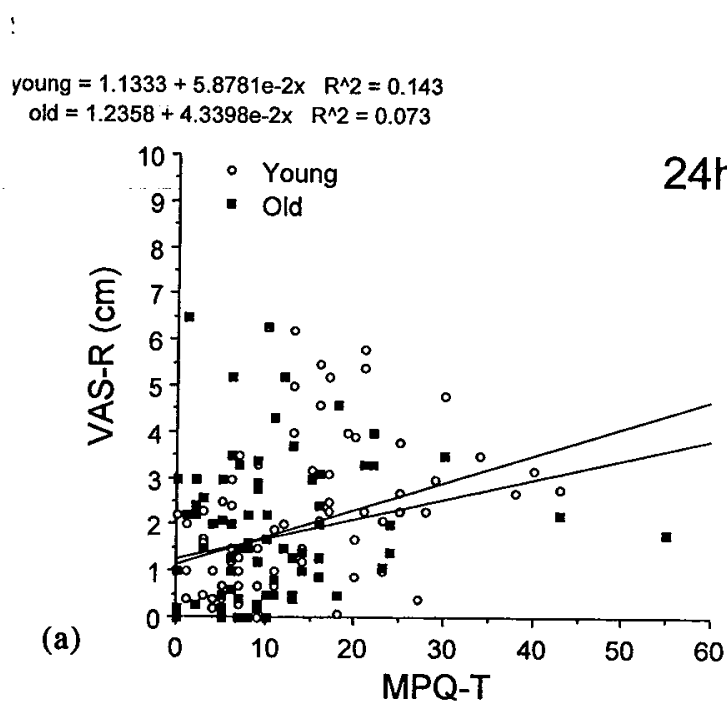

young $=1.9623+6.6875 x \quad R^{\wedge} 2=0.326$

old $=1.4469+6.4192 x \quad R^{\wedge} 2=0.327$

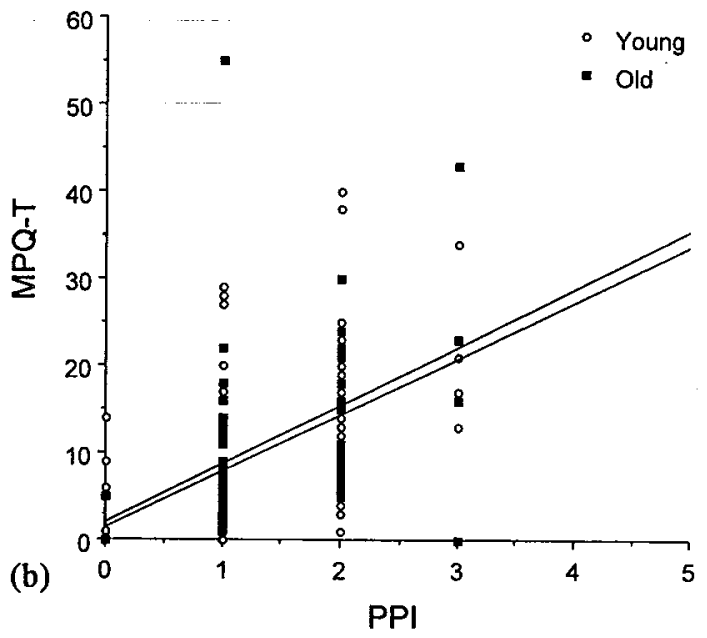

young $=0.44943+1.0215 x \quad R^{\wedge} 2=0.316$ old $=0.53896+0.92593 \times \quad R^{\wedge} 2=0.263 \quad 24 h$
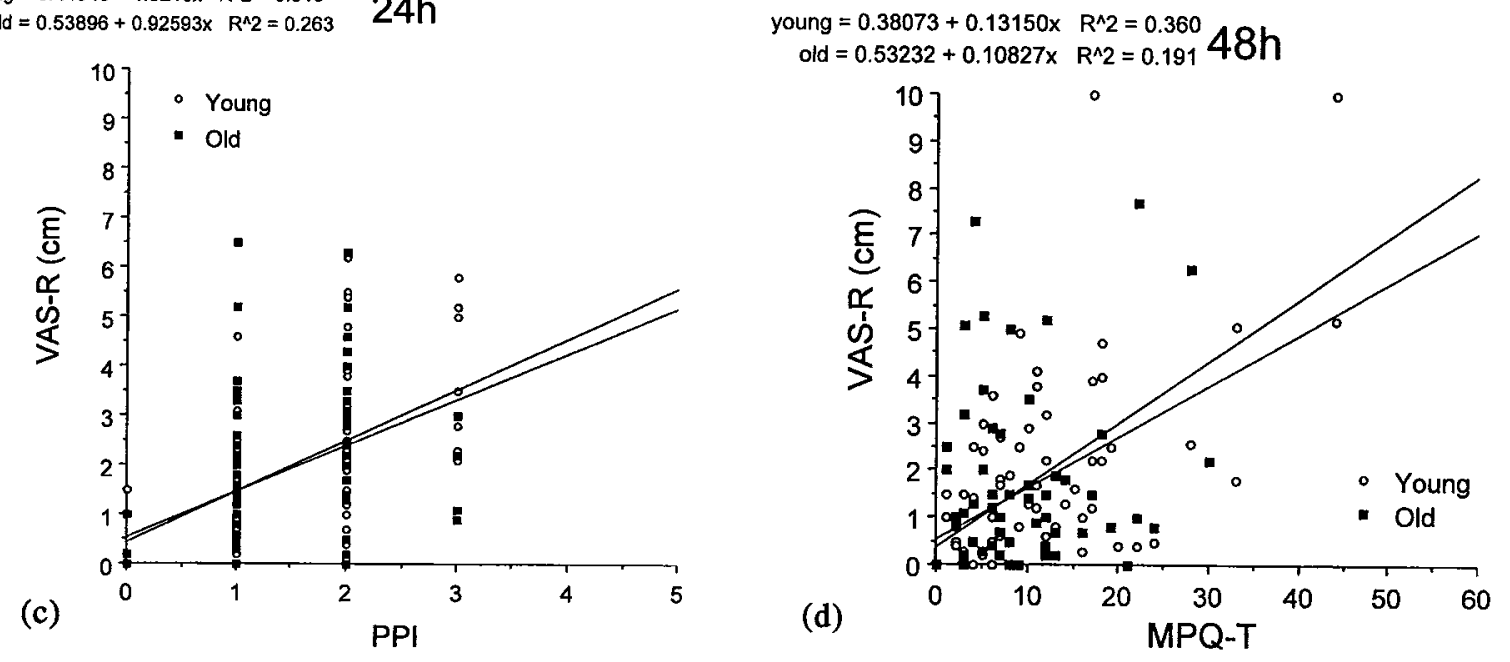

young $=7.1900 \mathrm{e}-2+1.2067 \times R^{\wedge} 2=0.471$

old $=0.17149+1.1484 x \quad R^{\wedge} 2=0.412$

$48 \mathrm{~h}$

young $=0.96801+6.0616 x \quad R^{\wedge} 2=0.571$
old $=1.0994+5.4678 \times \quad R^{\wedge} 2=0.572$$\quad 48 h$
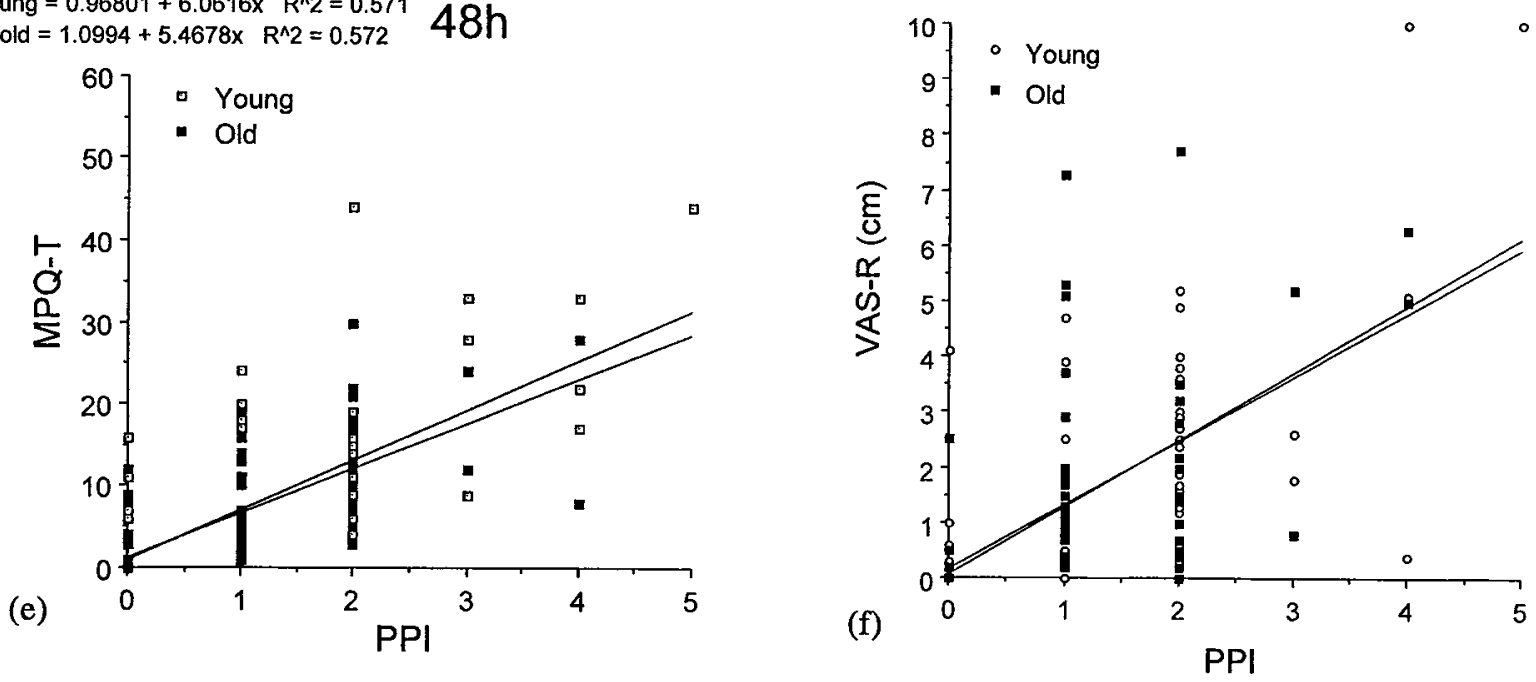

Fig. 5. (a-f) Scores on each pair of pain scales in younger and older patients on POD 1 and POD 2. The regression line and equation relating each pair of scales for each AGE is shown. The relationship between the two measures of intensity (VAS-R and PPI) did not differ between the age groups (c) and (f). (a) and (d) show the regression lines relating VAS-R and MPQ-T on POD $1(P \leq 0.05)$ and POD $2(P \leq 0.05)$ in the two age groups. 


\subsection{Age differences in PCA opioid use}

This is the first report of an interaction between age and postoperative day in the amount of opioid self-administered. We recently reported a similar trend in a sample made up of both male and female general surgery patients (Gagliese et al., 2000). An age-related decrease in opioid self-administration has been well-documented (Burns et al., 1989; Giuffre et al., 1991; Macintyre and Jarvis, 1995; Gagliese et al., 2000). This has been attributed to age-related increases in the analgesic efficacy of opioids (Kaiko, 1980; Moore et al., 1990) which may be secondary to agerelated changes in morphine metabolism (Owen et al., 1983; Baillie et al., 1989; Laizure et al., 1993; Van Crugten et al., 1997). Unfortunately, the current study did not assess morphine pharmacology, making it impossible to determine the role of this factor in the differences observed.

In both age groups, less morphine was consumed on POD 2 than POD 1, however, the decrease over time was greater in younger than older men. As a result, with time, the age groups became more similar. There may be several reasons for this interaction. It simply may result from a floor effect among the older patients which limits the magnitude of decrease over time. More interestingly, it may reffect age differences in the course of postoperative pain and recovery. Previous studies have suggested that the duration of postoperative pain may be greater in older than younger patients (Melzack et al., 1987; Gagliese et al., 2000). Consistent with this, following experimentally induced tissue damage, wound healing, hyperalgesia, and inflammation also are prolonged in older subjects (Ashcroft et al., 1995; Iwata et al., 1995; Zheng et al., 2000). These factors may contribute to the increased risk of chronic postsurgical pain with age (White et al., 1997). Future studies should assess the relationship between pain, PCA use, morphine pharmacology, wound healing, hyperalgesia, and recovery in younger and older surgical patients.

Although the age difference in morphine consumption may be small (18 mg over 2 days) and of uncertain clinical significance in and of itself, these data are clinically important because they provide further evidence that older patients are capable of utilizing PCA to obtain levels of analgesia comparable to (Gagliese et al., 2000) or better than younger patients. This is the first report of greater analgesia among older than younger patients using this modality. Elderly patients often have been excluded from PCA because of misconceptions regarding their ability and willingness to use the apparatus (Hofland, 1992; Pasero and McCaffery, 1996a,b). We have previously shown that attitudes towards PCA use are not age-related (Gagliese et al., 2000 ). The present data support our previous conclusion that age is not an impediment to effective usage of PCA (Gagliese et al., 2000).
4.2. Age differences in pain scores and in the psychometric properties of the pain scales

Age differences in pain scores were dependent on the scale used.- Specifically, pain scales comprising verbal descriptions of pain (MPQ, PPI) yielded different results than the 'non-verbal' intensity measure. On the verbal descriptor measures, older patients reported lower pain levels than younger patients whereas age differences were not found on the VAS.

One explanation of the discrepancy between the scales is that it reffects age differences in the psychometric properties of the scales rather than actual differences in pain. We found evidence for subtle differences in the convergent validity and sensitivity of the scales in younger and older patients. Although interscale correlations, a measure of convergent validity, were similar in the two groups, they were generally lower in the older group, with some failing to reach significance. Importantly, on both postoperative day, the correlation between VAS-R and MPQ scores was significantly lower in the older than younger group. The regression analyses further supported this conclusion. Again, only the regression line between VAS-R and the MPQ differed between the two groups. Therefore, the relationship between these two scales may be different in older and younger patients.

In both age groups, ratings on one scale often were not predictive of ratings on another scale. For instance, patients who indicated that their pain was 'distressing', a score of $3 / 5$ on the PPI, endorsed pain levels along the entire continuum of the VAS. This is an unexpected finding given that the scales are supposed to measure the same component of pain and were completed during the same assessment session, ruling out fluctuations in pain intensity over time and inadequate recall. A troubling explanation for the discordant ratings is the possibility of random responding on one or more of the scales. The moderate interscale correlations would argue against this. Another possibility is that the scales do not measure the same dimension of pain and so the seeming discrepancies are merely artefactual. It is impossible to draw conclusions regarding the reasons for the discordant responses. Future work is needed to address this issue.

Pain scale sensitivity, the ability to detect change over the two assessment times, did not differ between the scales or age groups suggesting that all three scales are appropriate for the assessment of change over time within an age group. However, the discrepancy between the scales for the effect of age suggests that the VAS is not sufficiently sensitive to detect age differences. A similar conclusion has been reached regarding the sensitivity of the VAS for the assessment of changes in chronic pain (Svensson, 1998). This suggests that the pattern of age differences in pain may reflect, in part, differences in the sensitivity of the scales. It is difficult to understand why the VAS would show sensitivity comparable to the other measures for the assessment 
of within-group effects but less sensitivity for cross-group comparisons. One possibility is that the effect of time is substantially larger than that of age, however the small effect sizes would argue against this.

These results suggest that age differences in postoperative pain are better captured by verbal descriptions than 'nonverbal' measures of intensity and that there are subtle differences in the relationship between measures of pain intensity and pain quality in younger and older men. In previous studies, increasing age has been associated with a higher frequency of incomplete or unscorable responses on the VAS (Kremer et al., 1981; Jensen et al., 1986; Gagliese and Melzack, 1997) but not on verbal descriptor scales (Jensen et al., 1986; Gagliese and Melzack, 1997) or the MPQ (Gagliese and Melzack, 1997). In the present study, only patients who were able to complete the scales were included in the analyses. In addition, the VAS may have poorer face validity than other scales for the elderly (Herr and Mobily, 1993; Benesh et al., 1997). Taken together, these data raise serious limitations for the use of the VAS in future studies of elderly populations.

\section{General conclusions}

A discrepancy between measures of pain qualities and intensity across age groups also has been reported in samples of people with chronic arthritis (Gagliese and Melzack, 1997) and heterogeneous chronic pain complaints (Gagliese and Melzack, 2002a,b). In these studies, the same pattern emerged whether pain intensity was assessed with the VAS, the verbal descriptor scale, the behavioural rating scale (Gagliese and Melzack, 1997), or the numeric rating scale (Gagliese and Melzack, 2002a,b). The results were also consistent whether the full (Gagliese and Melzack, 2002a,b) or short form (Gagliese and Melzack, 1997) of the MPQ was used to assess the qualities of chronic pain. It is interesting that in the current study, the PPI also differed between the age groups. This difference between the studies may reflect increased power to detect differences because the current sample is twice as large as the previous one (Gagliese and Melzack, 1997). On the other hand, it may reflect differences in postoperative versus chronic pain. In addition to extending this pattern to postoperative pain, this study is the first to include repeated measures. This allows for the assessment of scale sensitivity and shows that the discrepancy between scales is maintained over time.

Reasons why verbal descriptors but not abstract intensity ratings of pain may change with age should be studied further. In addition to psychometrics, it may reflect age differences in the use of pain language, an issue that has not received empirical attention. A related possibility is that completion of the scales requires different cognitive abilities that might vary across age groups. VAS ratings require quantification of pain intensity and abstract reasoning to determine the length of the line that corresponds to the intensity of pain (Ohnhaus and Adler, 1975; Wewers and Lowe, 1990). On the other hand, selection of verbal descriptors requires basic linguistic skills and the ability to identify the descriptor that best matches the intensity of pain (Ohnhaus and Adler, 1975). Completing the MPQ may be even more complex because choosing adjectives on this scale requires subtle differentiation of the qualities of experience; for instance hot versus burning sensations (Melzack, 1975). Although the cognitive and linguistic demands are different for these scales, it is not yet known how age might affect these processes.

More interestingly, the differences may have implications for our understanding of basic pain mechanisms. Melzack and Casey (1968) proposed that the different dimensions of pain may be subserved by different but interacting physiological substrates. Perhaps, the pattern of age differences in the qualities of pain reflects a differential effect of age on the integrity or activity levels of these systems. There is evidence that age-related changes in the neurobiological substrates of pain are not uniform throughout the central nervous system (Gagliese and Melzack, 2000). The implications of this pattern of age differences for basic pain mechanisms should be given serious empirical attention.

\section{Limitations and future directions}

There are several limitations of this study that must be considered in the interpretation of the results. The most important concerns the generalizability of the results. The older group had a mean age of only 67 years. As such, these results may not apply to significantly older elderly patients. In addition, only patients who could understand the use of PCA and who completed the pain scales were included in this study thus excluding acutely and chronically confused elderly patients. Although all patients were oriented at the time of assessment, we did not employ formal measures of cognition, raising the possibility that some of the patients experienced undetected confusion. However, excluding patients who were not able to complete the pain scales probably minimized this possibility. Therefore, the applicability of these results is limited to young-elderly, male, elective surgery patients with sufficient cognitive ability to understand the use of PCA and complete pain scales. Future research should examine these issues amongst the oldestold, women, and the cognitively impaired elderly. Another limitation of this study is that the order of pain scale completion was not counterbalanced. The order of scale completion was VAS-R, MPQ, PPI, and then VAS-M. This order was chosen so that patients would complete the static, at rest, pain measures before the dynamic measure (VAS-M) that could have inflated the static estimates. However, this may have inadvertently introduced a sequence or fatigue effect. Clearly, these results require replication with randomized order of scale completion, the inclusion of both horizontal and vertical VASs, numeric rating scales and pain thermo- 
meters, with frail elderly patients and with a variety of surgical procedures. We are currently conducting such a study. Until these more complete data are available, the best conclusion that can be drawn is that the assessment of postoperative pain across the adult life span should include verbal descriptor measures of the intensity of pain, such as the PPI, as well as measures of the qualities of pain, such as the MPQ.

\section{Acknowledgements}

We are grateful for the help provided by the staff of the Department of Anaesthesia, Department of Urology and the PACU at the Toronto General Hospital, Toronto, Ontario, Canada. The authors thank A. Ardiel Wowk and M. Jackson for their help with patient care and data collection, scoring, entry and checking. This research was supported in part by grant \# MCT-38144 from the Canadian Institutes of Health Research (CIHR) and a CIHR Investigator Award to J. Katz and a CIHR Postdoctoral Fellowship Award and University of Toronto Faculty of Medicine Dean's Fund Grant to L. Gagliese.

\section{References}

American Pain Society. Pain: the fifth vital sign. http://www.ampainsoc.org/advocacy/fifth.htm 1995

Anastasi A. Psychological testing. New York, NY: Macmillan, 1988.

Ashcroft GS, Horan MA, Ferguson MW. The effects of ageing on cutaneous wound healing in mammals. J Anat 1995;187:1-26.

Badaoui R, Riboulot M, Ernst C, Ossart M. L'analgésie postopératoire autocontrolée par le patient agé. Cahiers d'Anesthésiologie 1996;44:519-522.

Baillie SP, Bateman DN, Coates PE, Woodhouse KW. Age and the pharmacokinetics of morphine. Age Ageing 1989;18:258-262.

Bellville JW, Forrest WH, Miller E, Brown BW. Influence of age on pain relief from analgesics. J Am Med Assoc 1971;217:1835-1841.

Benesh LR, Szigeti E, Ferraro FR, Gullicks JN. Tools for assessing chronic pain in rural elderly women. Home Healthcare Nurse 1997;15:207-211.

Bennett-Guerrero E, Welsby I, Dunn TJ, Young LR, Wahl TA, Diers TL, Phillips-Bute BG, Newman MF, Mythen MG. The use of a postoperative morbidity survey to evaluate patients with prolonged hospitalization after routine, moderate-risk, elective surgery. Anesth Analg 1999;89:514-519.

Burns JW, Hodsman NBA, McLintock TTC, Gillies GWA, Kenny GNC, McArdle CS. The influence of patient characteristics on the requirements for postoperative analgesia. Anaesthesia 1989;44:2-6.

Cousins M. Acute and postoperative pain. In: Wall PD, Melzack R, editors. Textbook of pain, Edinburgh: Churchill Livingstone, 1994. pp. 284305 .

Duggleby W, Lander J. Cognitive status and postoperative pain: older adults. J Pain Symptom Manage 1994;9:19-27.

Dunn G. Design and analysis of reliability studies. New York, NY: Oxford University Press, 1989

Egan KJ, Ready LB. Patient satisfaction with intravenous PCA or epidural morphine. Can J Anaesth 1994;41:6-11.

Egbert AM, Parks LH, Short LM, Burnett ML. Randomized trial of postoperative patient-controlled analgesia vs intramuscular narcotics in frail elderly men. Arch Intern Med 1990;150:1897-1903.

Ergina PL, Gold SL, Meakins JL. Perioperative care of the elderly patient. World J Surg 1993;17:192-198.
Gagliese L. Assessment of pain in the elderly. In: Turk DC, Melzack R, editors. Handbook of pain assessment, New York, NY: Guilford Press, 2001. pp. 119-133.

Gagliese L, Jackson M, Ritvo P, Wowk A, Katz J. Age is not an impediment to effective use of patient controlled analgesia by surgical patients. Anesthesiology 2000;93:601-610.

Gagliese L, Melzack R. Age differences in the quality of chronic pain: a preliminary study. Pain Res Manage 1997;2:157-162.

Gagliese L, Melzack R. Age differences in nociception and pain behaviours in the rat. Neurosci Biobehav Rev 2000;24:843-854.

Gagliese L, Melzack R. Age-related differences in the qualities but not the intensity of chronic pain. Submitted for publication, 2002a

Gagliese L, Melzack R. The chronic pain centre patient: age differences and similarities in pain and psychological characteristics. Submitted for publication, 2002b.

Giuffre M, Asci J, Arnstein P, Wilkinson C. Postoperative joint replacement pain: description and opioid requirements. J Post Anesth Nurs 1991;6:239-245.

Herr KA, Mobily PR. Comparison of selected pain assessment tools for use with the elderly. Appl Nurs Res 1993;6:39-46.

Hofland SL. Elder beliefs: blocks to pain management. J Gerontol Nurs 1992;18:19-24.

Hunter JE, Schmidt FL. Methods of meta-analysis. Newbury Park, CA: Sage Publications, 1990.

Huskisson EC. Visual analogue scales. In: Melzack R, editor. Pain measurement and assessment, New York, NY: Raven Press, 1983. pp. 33-37.

Iwata K, Kanda K, Tsuboi Y, Kitajima K, Sumino R. Fos induction in the medullary dorsal horn and $\mathrm{C} 1$ segment of the spinal cord by acute inflammation in aged rats. Brain Res 1995;678:127-139.

Jensen MP. Validity of self-report and observation measures. In: Jensen TS, Turner JA, Wiesenfeld-Hallin Z, editors. Proceedings of the 8th World Congress on pain, vol. 8. Seattle, WA: IASP Press, 1997. pp. 637-662.

Jensen MP, Karoly P. Self-report scales and procedures for assessing pain in adults. In: Turk DC, Melzack R, editors. Handbook of pain assessment, New York, NY: Guilford Press, 1992. pp. 135-151.

Jensen MP, Karoly P. Self-report scales and procedures for assessing pain in adults. In: Turk DC, Melzack R, editors. Handbook of pain assessment, New York, NY: Guilford Press, 2001. pp. 15-34.

Jensen MP, Karoly P, Braver S. The measurement of clinical pain intensity: a comparison of six methods. Pain 1986;27:117-126.

Kaiko RF. Age and morphine analgesia in cancer patients with post-operative pain. Clin Pharmacol Ther 1980;28:823-826.

Katz J. Pain begets pain: predictors of long-term phantom limb pain and post-thoracotomy pain. Pain Forum 1997;6:140-144.

Kleinbaum DG, Kupper LL, Muller KE. Applied regression analysis and other multivariable methods. Boston, MA: PWS-KENT Publishing, 1988.

Kremer E, Atkinson JH, Ignelzi RJ. Measurement of pain: patient preference does not confound pain measurement. Pain 1981;10:241-249.

Laizure SC, Miller JH, Stevens RC, Donahue DJ, Laster RE, Brown D. The disposition and cerebrospinal fiuid penetration of morphine and its two major glucuronidated metabolites in adults undergoing lumbar myelogram. Pharmacotherapy 1993;13:471-475.

Lehmann KA. Patient-controlled intravenous analgesia for postoperative pain relief. In: Max M, Portenoy R, Laska E, editors. Advances in pain research and therapy, vol. 18. New York, NY: Raven Press, 1991. pp. 481-505.

Lewis B, Lewis D, Cumming G. Frequent measurement of chronic pain: an electronic diary and empirical findings. Pain 1995;60:341-347.

Lynch EP, Lazor MA, Gellis JE, Orav J, Goldman L, Marcantonio ER. The impact of postoperative pain on the development of postoperative delirium. Anesth Analg 1998;86:781-785.

Macintyre PE, Jarvis DA. Age is the best predictor of postoperative morphine requirements. Pain 1995;64:357-364.

Melzack R. The McGill Pain Questionnaire: major properties and scoring methods. Pain 1975;1:277-299. 
Melzack R, Abbott FV, Zackon W, Mulder DS, Davis MWL. Pain on a surgical ward: a survey of the duration and intensity of pain and the effectiveness of medication. Pain 1987;29:67-72.

Melzack R, Casey KL. Sensory, motivational and central control determinants of pain: a new conceptual model. In: Kenshalo D, editor. The skin senses, Springfield, IL: Charles C. Thomas, 1968. pp. 423-439.

Melzack R, Katz J. The McGill Pain Questionnaire: appraisal and current status. In: Turk DC, Melzack R, editors. Handbook of pain assessment, New York, NY: Guilford Press, 2001. pp. 35-52.

Miaskowski C, Crews J, Ready LB, Paul SM, Ginsberg B. Anesthesiabased pain services improve the quality of postoperative pain management. Pain 1999;80:23-29.

Moore AK, Vilderman S, Lubenskyi W, McCans J, Fox GS. Differences in epidural morphine requirements between elderly and young patients after abdominal surgery. Anesth Analg 1990;70:316-320.

Oberle K, Paul P, Wry J, Grace M. Pain, anxiety and analgesics: a comparative study of elderly and younger surgical patients. Can J Aging 1990;9:13-22.

Ohnhaus EE, Adler R. Methodological problems in the measurement of pain: a comparison between the verbal rating scale and the visual analogue scale. Pain 1975;1:379-384.

Owen JA, Sitar DS, Berger L, Brownell L, Duke PC, Mitenko PA. Agerelated morphine kinetics. Clin Pharmacol Ther 1983;34:364-368.

Pasero C, McCaffery M. Postoperative pain management in the elderly. In: Ferrell BR, Ferrell BA, editors. Pain in the elderly, Seattle, WA: IASP Press, 1996a. pp. 45-68.

Pasero CL, McCaffery M. Managing postoperative pain in the elderly. Am J Nurs 1996b;96:38-45.
Perry F, Parker RK, White PF, Clifford PA. Role of psychological factors in postoperative pain control and recovery with patient-controlled analgesia. Clin J Pain 1994;10:57-63.

Politser P, Schneidman D. American college of surgeons: socio-economic factbook for surgery 1990. Chicago, IL: American College of Surgeons, 1990.

Svensson E. Ordinal invariant measures for individual and group changes in ordered categorical data. Stat Med 1998;17:2923-2936.

Turk DC, Melzack R. Handbook of pain assessment. New York, NY: Guilford Press, 2001.

U.S. Department of Commerce. Global aging: comparative indicators and future trends. Washington, DC: U.S. Department of Commerce, Economics and Statistics Administration, Bureau of the Census, 1991.

Van Crugten JT, Somogyi AA, Nation RL, Reynolds G. The effect of old age on the disposition and antinociceptive response of morphine and morphine-6beta-glucoronide in the rat. Pain 1997;71:199-205.

Wewers ME, Lowe NK. A critical review of visual analogue scales in the measurement of clinical phenomena. Res Nurs Health 1990;13:227236.

White CL, LeFort SM, Amsel R, Jeans ME. Predictors of the development of chronic pain. Res Nurs Health 1997;20:309-318.

Wilke DJ, Savedra MC, Holzemer WL, Tesler MD, Paul SM. Use of the McGill Pain Questionnaire to measure pain: a meta-analysis. Nurs Res 1990;39:36-41.

Zheng Z, Gibson SJ, Khalil Z, Helme RD, McMeeken JM. Age-related differences in the time course of capsaicin-induced hyperalgesia. Pain 2000;85:51-58. 